\title{
Best Approximations in Cone Metric Spaces
}

\author{
Sh. ReZAPOUR
}

Abstract. Huang and Zhang defined cone metric spaces in 2007 ([1]). We shall give some results about characterization of best approximations in the cone metric spaces.

\section{INTRODUCTION}

Approximation theory has been investigated by many authors, and one can see the known book of Singer for a brief history of approximation theory ([8]). Recently, non-convex analysis has found some applications in optimization theory and approximation theory has special place in mathematics and so in non-convex analysis. In this way, Huang and Zhang defined the cone metric spaces in 2007 ([1]). At present, work on approximation theory is continued. For example, there are some works about best approximation, $\varepsilon$-best approximation and best simultaneous approximation in normed spaces, ordered normed spaces, 2-normed spaces and generalized 2-normed spaces ([2]-[7]).

Let $E$ be a real Banach space and $P$ a subset of $E$. $P$ is called a cone if (i) $P$ is closed, non-empty and $P \neq\{0\}$,

(ii) $a x+b y \in P$ for all $x, y \in P$ and all non-negative real numbers $a, b$, (iii) $P \cap(-P)=\{0\}$.

For a given cone $P \subseteq E$, we can define a partial ordering $\leq{ }_{P}$ with respect to $P$ by $x \leq_{P} y$ if and only if $y-x \in P$. In what follows we omit the index $P$ and write everywhere $\leq$ instead of $\leq_{P} . x<y$ will stand for $x \leq y$ and $x \neq y$, while $x \ll y$ will stand for $y-x \in$ int $P$, where int $P$ denotes the interior of $P$.

The cone $P$ is called normal if there is a number $K>0$ such that $0 \leq x \leq y$ implies $\|x\| \leq K\|y\|$, for all $x, y \in E$.

In the following we always suppose that $E$ is a Banach space, $P$ is a cone in $E$ with int $P \neq \emptyset$ and $\leq$ is partial ordering with respect to $P$.

Definition 1.1. Let $X$ be a non-empty set, $E$ a Banach space and $P$ a cone in $E$. Suppose the mapping $d: X \times X \rightarrow E$ satisfies

$\left(d_{1}\right) 0 \leq d(x, y)$ for all $x, y \in X$ and $d(x, y)=0$ if and only if $x=y$,

$\left(d_{2}\right) d(x, y)=d(y, x)$ for all $x, y \in X$,

$\left(d_{3}\right) d(x, y) \leq d(x, z)+d(z, y)$ for all $x, y, z \in X$.

2000 Mathematics Subject Classification. Primary: 41A65.

Key words and phrases. Cone metric space, Best approximation, Quasi Chebyshev. 
Then $d$ is called a cone metric on $X$, and $(X, d)$ is called a cone metric space ([1]).

Example 1.2. Let $E=\mathbb{R}^{2}, P=\{(x, y) \in E: x, y \geq 0\}, X=\mathbb{R}$ and $d: X \times X \rightarrow$ $E$ defined by $d(x, y)=(|x-y|, \alpha|x-y|)$, where $\alpha \geq 0$ is a constant. Then $(X, d)$ is a cone metric space $([1])$.

Example 1.3. Let $E=\ell^{1}, P=\left\{\left\{x_{n}\right\}_{n \in \mathbb{N}} \in E: x_{n} \geq 0\right.$, for all $\left.\mathrm{n}\right\},(X, \rho)$ a metric space and $d: X \times X \rightarrow E$ defined by $d(x, y)=\left\{\frac{\rho(x, y)}{2^{n}}\right\}_{n \in \mathbb{N}}$. Then $(X, d)$ is a cone metric space.

Definition 1.4. ([1]) Let $(X, d)$ be a cone metric space, $x \in X$ and $\left\{x_{n}\right\}_{n \in \mathbb{N}}$ a sequence in $X$. Then

(i) $\left\{x_{n}\right\}_{n \in \mathbb{N}}$ converges to $x$ whenever for every $c \in E$ with $0 \ll c$ there is a natural number $N$ such that $d\left(x_{n}, x\right) \ll c$ for all $n \geq N$. We denote this by $\lim _{n \rightarrow \infty} x_{n}=x$ or $x_{n} \rightarrow x$.

(ii) $\left\{x_{n}\right\}_{n \in \mathbb{N}}$ is a Cauchy sequence whenever for every $c \in E$ with $0 \ll c$ there is a natural number $N$ such that $d\left(x_{n}, x_{m}\right) \ll c$ for all $n, m \geq N$.

(iii) $(X, d)$ is a complete cone metric space if every Cauchy sequence is convergent.

Here we point to some elementary results of [1]. Let $(X, d)$ be a cone metric space, $P$ a normal cone with normal constant $K, x \in X$ and $\left\{x_{n}\right\}_{n \in \mathbb{N}}$ a sequence in $X$. Then

(i) $\left\{x_{n}\right\}_{n \in \mathbb{N}}$ converges to $x$ if and only if $d\left(x_{n}, x\right) \rightarrow 0$.

(ii) Limit point of every sequence is unique.

(iii) Every convergent sequence is Cauchy.

(iv) $\left\{x_{n}\right\}_{n \in \mathbb{N}}$ is a Cauchy sequence if and only if $d\left(x_{n}, x_{m}\right) \rightarrow 0$ as $n, m \rightarrow \infty$.

(v) If $x_{n} \rightarrow x$ and $x_{n}^{\prime} \rightarrow x^{\prime}$, then $d\left(x_{n}, x_{n}^{\prime}\right) \rightarrow d\left(x, x^{\prime}\right)$ as $n \rightarrow \infty$.

Definition 1.5. Let $(X, d)$ be a cone metric space and $B \subseteq X$. If every sequence in $B$ has a convergent subsequence to an element of $B$, then $B$ is called a sequentially compact subset of $X$.

Definition 1.6. Let $(X, d)$ be a cone metric space, $G$ a non-empty subset of $X$ and $x \in X$. We say that $g_{0} \in G$ is a best approximation of $x$ whenever $d\left(x, g_{0}\right) \leq d(x, g)$ for all $g \in G$. Then, we denote the set of all best approximations of $x$ in $G$ by $P_{G}(x)$.

Definition 1.7. Let $(X, d)$ be a cone metric space and $G$ a non-empty subset of $X$. We say that $G$ is a Chebyshev subset of $X$ if $P_{G}(x)$ is a singleton subset of $G$ for all $x \in X$. Also, we say that $G$ is a quasi Chebyshev subset of $X$ if $P_{G}(x)$ is sequentially compact subset of $X$ for all $x \in X$.

Definition 1.8. Let $X$ be a real vector space, $(X, d)$ a cone metric space and $G$ a non-empty subset of $X$. We say that $G$ is a pseudo Chebyshev subset of $X$ if there is no $x \in X$ such that $P_{G}(x)$ contains infinitely many linearly independent elements. 
Example 1.9. Let $E=\ell^{1}, P=\left\{\left\{x_{n}\right\}_{n \in \mathbb{N}} \in E: x_{n} \geq 0\right.$, for all $\left.n\right\},(X,\|\|$.$) a$ normed space, $G$ a quasi Chebyshev subset of $X$ and $d: X \times X \rightarrow E$ defined by $d(x, y)=\left\{\frac{\|x-y\|}{2^{n}}\right\}_{n \in \mathbb{N}}$. Then, $G$ is a quasi Chebyshev subset of $(X, d)$.

\section{Main Results}

Now we are ready to state our main results.

Lemma 2.1. Let $(X, d)$ be a cone metric space, $G$ a non-empty subset of $X$, $g_{0} \in G$ and $x \in X$. Then, $g_{0} \in P_{G}(x)$ if and only if there exists a function $f: X \rightarrow E$ such that $f\left(g_{0}\right)=d\left(x, g_{0}\right), f_{g_{0}}(g):=f(g)-f\left(g_{0}\right) \in P$ and $f_{d}(g):=$ $d(x, g)-f(g) \in P$ for all $g \in G$.

Proof. First suppose that there exists a function $f: X \rightarrow E$ such that $f\left(g_{0}\right)=$ $d\left(x, g_{0}\right), f_{g_{0}}(g) \in P$ and $f_{d}(g) \in P$ for all $g \in G$. Since $f_{g_{0}}(G) \subseteq P$ and $f_{d}(G) \subseteq P$, $f\left(g_{0}\right) \leq f(g)$ and $f(g) \leq d(x, g)$ for all $g \in G$. Thus, $d\left(x, g_{0}\right)=f\left(g_{0}\right) \leq f(g) \leq$ $d(x, g)$ for all $g \in G$. Hence, $g_{0} \in P_{G}(x)$.

For the converse part, define $f: X \rightarrow E$ by $f(t)=d(x, t)$. Then, $f\left(g_{0}\right)=d\left(x, g_{0}\right)$, $f_{g_{0}}(G) \subseteq P$ and $f_{d}(G)=\{0\} \subseteq P$.

Theorem 2.2. Let $(X, d)$ be a cone metric space, $G$ a non-empty subset of $X$ and $x \in X$. Then, $M \subseteq P_{G}(x)$ if and only if there exists a function $f: X \rightarrow E$ such that $f(m)=d(x, m), f_{m}(G) \subseteq P$ and $f_{d}(G) \subseteq P$ for all $m \in M$.

Proof. First suppose that there is a function $f: X \rightarrow E$ such that $f(m)=d(x, m)$, $f_{m}(G) \subseteq P$ and $f_{d}(G) \subseteq P$ for all $m \in M$. Then by Lemma 2.1, $m \in P_{G}(x)$ for all $m \in M$. Hence, $M \subseteq P_{G}(x)$.

For the converse part, take an arbitrary element $m_{1} \in M$. By Lemma 2.1, there is a function $f: X \rightarrow E$ such that $f\left(m_{1}\right)=d\left(x, m_{1}\right), f_{m_{1}}(G) \subseteq P$ and $f_{d}(G) \subseteq P$. Let $m \in M$. Then, $f_{m_{1}}(m) \in P$ and $f_{d}(m) \in P$. Since $m \in P_{G}(x), d(x, m) \leq$ $d\left(x, m_{1}\right) \leq f(m) \leq d(x, m)$. Hence, $f(m)=d(x, m)$. Also,

$$
f_{m}(g)=f(g)-f(m)=f(g)-d(x, m)=f(g)-d\left(x, m_{1}\right)=f_{m_{1}}(g) \in P
$$

for all $g \in G$. Therefore, $f$ is the desired function.

Corollary 2.3. Let $(X, d)$ be a cone metric space and $G \subseteq X$. Then, $G$ is a Chebyshev subset of $X$ if and only if there don't exist $x \in X$, distinct elements $g_{1}, g_{2} \in G$ and a function $f: X \rightarrow E$ such that $f\left(g_{i}\right)=d\left(x, g_{i}\right), f_{g_{i}}(G) \subseteq P$ and $f_{d}(G) \subseteq P$ for $i=1,2$.

Theorem 2.4. Let $(X, d)$ be a cone metric space and $G \subseteq X$. Then, $G$ is quasi Chebyshev subset of $X$ if and only if there don't exist $x \in X$, a sequence $\left\{g_{n}\right\}_{n \in \mathbb{N}}$ in $G$ without a convergent subsequence and a function $f: X \rightarrow E$ such that $f\left(g_{n}\right)=d\left(x, g_{n}\right), f_{g_{n}}(G) \subseteq P$ and $f_{d}(G) \subseteq P$ for all $n \in \mathbb{N}$.

Proof. First suppose that there exist $x \in X$, a sequence $\left\{g_{n}\right\}_{n \in \mathbb{N}}$ in $G$ without a convergent subsequence and a function $f: X \rightarrow E$ such that $f\left(g_{n}\right)=d\left(x, g_{n}\right)$, $f_{g_{n}}(G) \subseteq P$ and $f_{d}(G) \subseteq P$ for all $n \in \mathbb{N}$. Then by Theorem $2.2, g_{n} \in P_{G}(x)$ for 
all $n \in \mathbb{N}$. It follows that $P_{G}(x)$ is not sequentially compact.

For the converse part, suppose that $G$ is not quasi Chebyshev subset of $X$. Then, there exist $x \in X$ and a sequence $\left\{g_{n}\right\}_{n \in \mathbb{N}}$ in $P_{G}(x)$ without a convergent subsequence. By Theorem 2.2, there exists $f: X \rightarrow E$ such that $f\left(g_{n}\right)=d\left(x, g_{n}\right)$, $f_{g_{n}}(G) \subseteq P$ and $f_{d}(G) \subseteq P$ for all $n \in \mathbb{N}$.

The proof of the following Theorem is similar to that of Theorem 2.4.

Theorem 2.5. Let $X$ be a real vector space, $(X, d)$ a cone metric space and $G \subseteq X$. Then, $G$ is pseudo Chebyshev subset of $X$ if and only if there don't exist $x \in X$, infinitely many linearly independent elements $\left\{g_{n}\right\}_{n \in \mathbb{N}}$ in $G$ and a function $f: X \rightarrow E$ such that $f\left(g_{n}\right)=d\left(x, g_{n}\right), f_{g_{n}}(G) \subseteq P$ and $f_{d}(G) \subseteq P$ for all $n \in \mathbb{N}$.

\section{REFERENCES}

[1] Huang Long-Guang, Zhang Xian, Cone metric spaces and fixed point theorems of contractive mappings, J. Math. Anal. Appl., 332 (2007), 1468-1476.

[2] H. Mohebi, H. Sadeghi, A.M. Rubinov, Best approximation in a class of normed spaces with star-shaped cone, Numer. Funct. Anal. Optim., 27 (2006), no. 3-4, 411-436.

[3] H. Mohebi, H. Sadeghi, Best approximation in a class of ordered normed spaces without a pre-order relation, Int. J. Pure Appl. Math., 21 (2005), no. 2, 199-221.

[4] H. Mohebi, Downward sets and their best simultaneous approximation properties with applications, Numer. Funct. Anal. Optim., 25 (2004), no. 7-8, 685-705.

[5] Sh. Rezapour, Proximinal Subspaces of 2-normed Spaces, Anal. Theory Appl., Vol., 22, No. 2 (2006), 114-119.

[6] Sh. Rezapour, 1-type Pseudo-Chebyshev Subspaces in Generalized 2-normed Spaces, Australian J. Math. Anal. Appl., Vol. 4, No. 1 (2007), 1-7.

[7] Sh. Rezapour, $\varepsilon$-weakly Chebyshev Subspaces of Banach Spaces, Anal. Theory Appl., Vol. 19, No. 2 (2004), 130-135.

[8] I. Singer, Best approximation in normed linear spaces by elements of linear subspaces, Springer-Verlag, Berlin, 1970.

SH. REZAPOUR

Department of Mathematics

Azarbaidjan University of Tarbiat Moallem AZARSHAHR, TABRIZ

IRAN

E-mail address: sh.rezapour@azaruniv.edu 